\title{
Conceptual Understanding through Efficient Automated Design of Quantum Optical Experiments
}

\author{
Mario Krenn, 1,2,3,4,* Jakob S. Kottmann, ${ }^{1,2}$ Nora Tischler, ${ }^{5,6}$ and Alán Aspuru-Guzik ${ }^{1,2,3,7, \dagger}$ \\ ${ }^{1}$ Chemical Physics Theory Group, Department of Chemistry, University of Toronto, Canada \\ ${ }^{2}$ Department of Computer Science, University of Toronto, Canada \\ ${ }^{3}$ Vector Institute for Artificial Intelligence, Toronto, Canada \\ ${ }^{4}$ Institute of Advanced Research in Artificial Intelligence (IARAI), Vienna, Austria \\ ${ }^{5}$ Centre for Quantum Computation and Communication Technology (Australian Research Council), \\ Centre for Quantum Dynamics, Griffith University, Brisbane, Australia \\ ${ }^{6}$ Dahlem Center for Complex Quantum Systems, Freie Universität Berlin, Germany \\ ${ }^{7}$ Lebovic Fellow, Canadian Institute for Advanced Research (CIFAR), Toronto, Canada
}

(Received 23 November 2020; revised 1 July 2021; accepted 1 July 2021; published 26 August 2021)

Artificial intelligence (AI) is a potentially disruptive tool for physics and science in general. One crucial question is how this technology can contribute at a conceptual level to help acquire new scientific understanding. Scientists have used AI techniques to rediscover previously known concepts. So far, no examples of that kind have been reported that are applied to open problems for getting new scientific concepts and ideas. Here, we present THESEUs, an algorithm that can provide new conceptual understanding, and we demonstrate its applications in the field of experimental quantum optics. To do so, we make four crucial contributions. (i) We introduce a graph-based representation of quantum optical experiments that can be interpreted and used algorithmically. (ii) We develop an automated design approach for new quantum experiments, which is orders of magnitude faster than the best previous algorithms at concrete design tasks for experimental configuration. (iii) We solve several crucial open questions in experimental quantum optics which involve practical blueprints of resource states in photonic quantum technology and quantum states and transformations that allow for new foundational quantum experiments. Finally, and most importantly, (iv) the interpretable representation and enormous speed-up allow us to produce solutions that a human scientist can interpret and gain new scientific concepts from outright. We anticipate that THESEUS will become an essential tool in quantum optics for developing new experiments and photonic hardware. It can further be generalized to answer open questions and provide new concepts in a large number of other quantum physical questions beyond quantum optical experiments. THESEus is a demonstration of explainable AI (XAI) in physics that shows how AI algorithms can contribute to science on a conceptual level.

DOI: 10.1103/PhysRevX.11.031044

\section{INTRODUCTION}

Photons are at the core of many quantum technologies that promise advances for imaging applications [1], efficient metrological schemes [2], and fundamentally secure communication protocols [3] as well as simulation [4] and computation techniques [5-7] that are beyond the capabilities of their classical counterparts. Besides, photons are

\footnotetext{
*mario.krenn@utoronto.ca

†alan@aspuru.com
}

Published by the American Physical Society under the terms of the Creative Commons Attribution 4.0 International license. Further distribution of this work must maintain attribution to the author(s) and the published article's title, journal citation, and DOI.
Subject Areas: Computational Physics,

Quantum Physics, Quantum Information also among the core players in the experimental investigation of fundamental questions about the local and realistic nature of our Universe.

Motivated by these opportunities, recent years have seen dramatic advances in quantum optical technology, which include highly complex operations in integrated photonic chips [8-12], generation of complex multiphotonic entanglement and its application [13-15], and the development and application of high-quality deterministic single-photon emitters [16] and highly efficient photon-number-resolving detectors [3,17].

To advance technological and fundamental progress further and to enable the exploration of numerous proposed ideas in laboratories, new experimental concepts and ideas are instrumental. Frequently, however, the design of experimental setups even for well-defined targets is challenging for 
the intuition of human experts, and existing systematic schemes (e.g., Ref. [18]) to date provide solutions only for specific experimental scenarios. For that reason, computational design methods for quantum optical experiments have been introduced [19], in the form of topological search augmented with machine learning [20,21], genetic algorithms [22,23], active learning approaches [24], and optimization of parametrized setups [25]. Unfortunately, due to the complexity and size of the Hilbert space as well as the breadth of quantum optical applications, those algorithms may have severe drawbacks, such as inefficient discovery rates, requirements of a huge amount of training data, or specialization on narrow sets of problems. Most importantly, to the best of our knowledge, no method so far has shown how to systematically extract new scientific ideas, concepts, and understanding from the solutions of the computer algorithm.

Here, we demonstrate THESEUS, an automated design algorithm for quantum optics with highly interpretable representation that allows scientists to understand solutions quickly. THESEUS is generally applicable to discretevariable quantum optics problems (including postselected, nonpostselected, and heralded states and probabilistic and deterministic photon sources), does not need training data, and is orders of magnitude faster than previous comparable approaches. An essential feature of THESEUs is the possibility to simplify the solution to its conceptual cores. Physicists can then interpret, understand, and generalize the underlying ideas and concepts. These advances allow us to apply THESEUS to solve several previously open questions about quantum experiments, which we briefly summarize now.

Concretely, we investigate complex multiphotonic entanglement and its applications. We discover a concept to generate maximally entangled high-dimensional multiparticle quantum states. Whether these states can even be created with the given (experimentally feasible) resources has been questioned in the past. Instead of finding a solution with perfect fidelity, the algorithm discovers a way to suppress undesired terms in the quantum state to arbitrary small probabilities. The idea can be understood combinatorially and can be generalized to many other cases.

Furthermore, we discover a new idea that allows for the generation of heralded entanglement, which is crucial in protocols for quantum communication and photonic quantum computation. Here, the new enabling concept is the destructive interference of vacuum contributions in the quantum state. A vacuum contribution is a situation where no photons emerge from the setup. The algorithm finds a way to construct such a situation twice in the same setup, but with opposite sign, such that they cancel each other. Therefore, the experiment creates the correct photon state.

In addition, we gain new insights into complex photonic quantum transformations and their generalizations to higher dimensions - a significant task that is not yet well understood. We gain an understanding of how heralded photonic CNOTs work in terms of an intuitive combinatorial picture.. Here, individual terms in the quantum state (edges of the graph) can block or unblock other terms depending on their mode number. Concretely, the amplitude of blocked terms is significantly reduced as a consequence of the probabilistic photon-pair sources.

In all of these cases, we uncover previously unknown generalizable patterns and new experimental ideas and interpretations.

THESEUS contributes directly to artificial scientific discovery, a field which-loosely put-studies how far science can be automated. Exciting early contributions involve automated genetic experiments [26] and the extraction of equation of motions from experimental data [27]. In recent years, fueled by massive growth of computing power and algorithmic advances such as deep learning, many more scientific tasks have been successfully automated in various domains. Examples involve the design and laboratory automation in chemistry [28-30], nanophotonics [31,32], quantum circuit design [33,34], and many others (for more details, see Ref. [19]).

An important question now is how new scientific concepts and understanding can be automatically discovered. Here is where our approach differs significantly from others that try to employ machine learning to extract scientific concepts. The main difference is that these methods so far have been successfully applied only to rediscover previously known concepts [35], and generalization to the much more challenging task of discovering new concepts has not been achieved yet. Examples include the identification of astronomical concepts such as the heliocentric world view which has already been considered by Copernicus [36], the arrow of time and related thermodynamical concepts that were discovered in the 20th century [37], the identification of certain interferometric devices that have been used by optical physicists for many years [24], or the extraction of symbolic equations for various physical systems [38]. Those are significant works that indicate great future possibility. However, they come with a grain of salt: It is not clear how much prior knowledge the scientists implicitly use to identify those concepts from the computer algorithms. Therefore, it is unclear whether these methods can be extended to actual open questions.

In quantum optics, in two works new concepts have been identified $[39,40]$ using a topological search algorithm [20]. There, tens of thousands of CPU hours are necessary to arrive at a useful design. Those solutions are represented directly as a sequence of optical elements, which are very unintuitive to interpret conceptually. Moreover, the sequences are highly nonoptimized, because they emerge through random processes. As a consequence, it requires scientists weeks or even months to understand the underlying principles (see Ref. [19] for more details). 
In contrast to those previous approaches, we introduce for the first time an algorithm that produces solutions that can be interpreted and rationalized by scientists. The interpretable solutions allow human scientists to understand the new, underlying concepts in quasi-real time. We explicitly demonstrate this feature by solving several previously unresolved questions. In all of those cases, we can interpret and understand the underlying design concepts outright. Put differently, THESEUS is an algorithm that can provide targeted and systematic new conceptual understanding in a scientific domain. We believe, therefore, that THESEUS is an important step toward the goal of interpretable and explainable artificial intelligence in science that will assist human researchers at a conceptual level.

\section{RESULTS}

\section{A. Graph theory-quantum experiment mapping}

Weighted colored graphs (explained in Fig. 1) can encode the information produced by a photonic quantum experiment. The vertices correspond to spatial photon paths, and edges between vertex $v_{1}$ and $v_{2}$ stand for probabilistic photon pairs in path $v_{1}$ and $v_{2}$. The edge color represents the internal mode number of the photons, and edge weights $\omega$ stand for amplitudes. Previously, the description was applicable only to postselected experiments involving probabilistic photonpair sources [41] and linear optical components [42].

We significantly extend the abstract description of quantum optics experiments as colored weighted graphs, demonstrating how general quantum optics technology and questions can be raised and solved using the new framework. The extensions allow us for the first time to use the framework of weighted colored graphs for computational design of quantum optical experiments and hardware.

Specifically, here, we introduce a weight function $\Phi(\omega)$ that gives access to the complete information of quantum optical experiments (rather than only postselected states, as in Refs. $[41,42,45]$ ) and allows us to generalize the scope of the method significantly. It allows for the description of nonpostselected states, triggered and heralded photonic states, states with multiple excitations per mode (such as NOON states), and general quantum transformations. Furthermore, it enables the description of photonnumber-sensitive and -insensitive detectors [which correspond to different projections of $\Phi(\omega)]$ and deterministic photon sources such as quantum dots (see Appendix A for details).

We emphasize explicitly that the weight function $\Phi(\omega)$ is a crucial conceptual advance that is necessary for all results presented in our manuscript. The weight function directly connects the experimental setup with the quantum state. It (a)

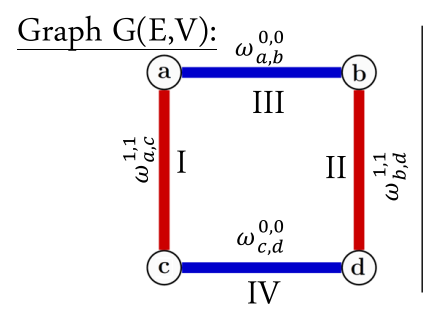

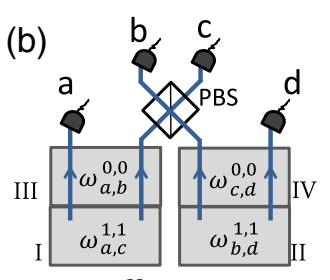

Bulk optics

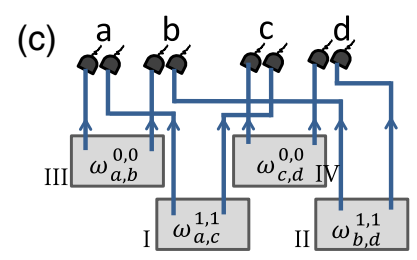

Integrated photonics

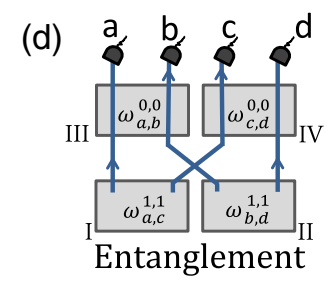

by path identity

$$
\begin{gathered}
\text { Weight function } \\
\Phi(\omega) \approx \sum_{m} 1 / m !\left(\sum_{E(G)} \omega(E) x^{\dagger}(E) y^{\dagger}(E)\right)^{m}=\sum_{m} 1 / m !\left(\omega_{a, b}^{0,0} a_{0}^{\dagger} b_{0}^{\dagger}+\omega_{c, d}^{0,0} c_{0}^{\dagger} d_{0}^{\dagger}+\omega_{a, c}^{1,1} a_{1}^{\dagger} c_{1}^{\dagger}+\omega_{b, d}^{1,1} b_{1}^{\dagger} d_{1}^{\dagger}\right)^{m} \\
\begin{array}{c}
\text { Applying on vacuum: } \\
|\psi\rangle=\Phi(\omega) \mid \text { vac }\rangle
\end{array}
\end{gathered}
$$

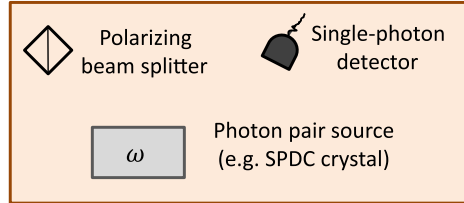

FIG. 1. A weighted edge-colored graph as an abstract and efficient representation of the quantum information of a large variety of quantum optics experiments. (a) As a specific example, we show a graph with four vertices and four colored and weighted edges. The vertices $a-d$ correspond to photonic paths, the edges correspond to correlated photon pairs, the edge colors stand for mode numbers, and weights $\omega \in \mathbb{C}$ stand for complex coefficients. Probabilistic sources create the photon pairs (edges). Thus, the entire information about the quantum state is represented by $\Phi(\omega)$, with $x_{k}^{\dagger}$ being a creation operator of a photon in path $x$ with mode number $k$. The graph can directly be translated into a number of different quantum optical technologies, such as (b) standard bulk optics, for example, with polarization encoding, or (c) path encoding as the carrier of quantum information or (d) entanglement by path identity. In all examples, the gray squares I-IV stand for probabilistic pair sources (such as nonlinear crystals which create photon pairs using spontaneous parametric down-conversion). Two crystals in a row, such as in (b), is a standard tool for generating photonic entanglement, denoted cross-crystal sources $[43,44]$. Two of the four crystals produce a photon pair. Blue lines show the path of the photon pair from the crystal to the photon detectors $a-d$. We show a number of examples in the Appendix A and provide an open-source code for the translation. The results of the quantum experiments can directly be calculated from the information of the graph. For example, a prominent technique is to condition the state on detecting a photon in each of the four detectors (postselection). The equivalent formulation in terms of graphs is the sum of all subsets of edges that contain every vertex exactly once, which is called perfect matching. It reduces the example quantum state to two terms. If all weights are equal, the resulting quantum state is a four-qubit GHZ state. Access to $\Phi(\omega)$ allows for the optimization of nonpostselected, heralded, and triggered quantum states, too, as we show in examples within the manuscript. 


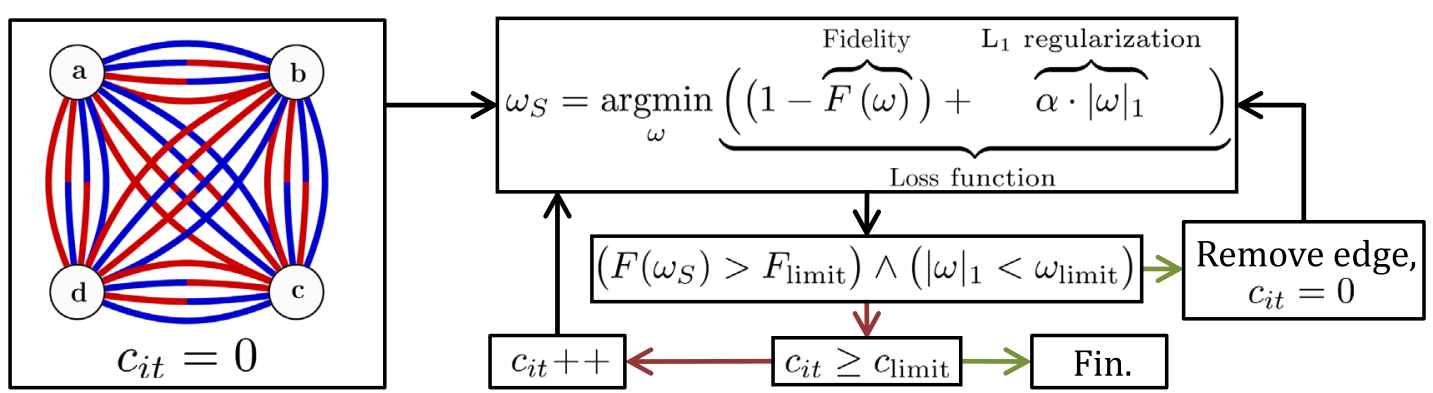

FIG. 2. Algorithm-Theseus: The initial graph contains all possible edges between each vertex, leading to $|G|=d^{2}[n(n-1) / 2]$ edges (with $n$ vertices and $d$ different edge colors), each of them having an independent complex weight $\omega_{v_{1}, v_{2}}^{m_{1}, m_{2}}$, where $v_{1}\left(v_{2}\right)$ stands for the first (second) vertex, and $m_{1}\left(m_{2}\right)$ stands for the color of the edge close to vertex $v_{1}\left(v_{2}\right)$.. The main step is a minimization of the loss function, which contains the quantum fidelity in terms of weights of the graph. Additionally, an $L_{1}$ regularization term controls the magnitude of the weights. If the weights identified by the optimization, $\omega_{S}$, lead to fidelities larger than $F_{\text {limit }}$, and the magnitude of the weights is smaller than $\omega_{\text {limit }}$, then one edge of the graph is removed, and the optimization continues with the smaller graph. On the other hand, if the criteria are not fulfilled, the same graph is optimized (with different starting conditions) until the discovery of a suitable solution or the number of iterations exceeds $c_{\text {limit }}$. The result of THESEUs is a weighted graph that leads to sufficiently large fidelities, with a small number of edges. This topological optimization enables the scientific interpretation and understanding of results.

can be constructed simply by enumerating all edges in the graph, and the quantum state can be obtained by applying the weight function (which is a function of operators) to the vacuum state, i.e.,

$$
|\psi\rangle=\Phi(\omega)|\mathrm{vac}\rangle .
$$

In this way, we obtain the quantum state (and all properties that can be computed from it) as a function of weights of the graph and, simultaneously, the quantum experimental setup. This fact is crucial, because now we can perform continuous, gradient-based optimization (similar to highly efficient optimization techniques used for training large neural networks) of the quantum state's properties. The result is a weighted graph that we can translate to quantum optical hardware implementation. With that, the weight function $\Phi(\omega)$ directly connects the quantum state and its properties with an experimental setup.

Moreover, we introduce here how graphs can be directly translated to several different schemes of photonic quantum optics, such as standard bulk optics [46], integrated photonics $[8,47]$ or entanglement by path identity $[39,48]$. A given graph can be translated in multiple ways to quantum experimental setups, while each setup corresponds to precisely one graph (more details in Appendix A). Thereby, our algorithm is not restricted to the relatively new idea of path identity which is experimentally just being explored $[48,49]$, but the experiments can be performed with well-established photonic technology. These novel features are necessary to use the graphtheoretical description as a tool for the automated design of quantum experiments that are feasible in state-of-the-art quantum photonics laboratories. We provide an opensource code for the translation between graphs and different types of quantum optical implementations.

\section{B. Graph-based automated design of quantum experiments}

The abstract and general representation of quantum experiments as graphs allows us to find a new method for automated designing quantum experiments. This possibility arises because we have a way to write the whole quantum state generated by an experimental setup as a function that depends only on the weights $\omega$ of the graph. Consequently, every function that we can derive from the quantum state is also a function that depends only on the weights $\omega$. Therefore, it is possible to write optimization objective functions in terms of weights $\omega$ of the graph.

For example, if we aim to find an experimental setup that produces a specific quantum state, the objective function is the state fidelity in terms of the weights of the graph. If we aim to find a transformation, then the objective function is the gate fidelity. Importantly, one can use the same technique for more general optimization targets, where neither the quantum state nor the quantum experiments are known beforehand. Examples are quantum metrology, where the objective function would be the Fisher information (written in terms of weights $\omega$ ), or quantum-enhanced imaging technologies, where the objective function could be a signal-to-noise ratio (again, in terms of weights $\omega$ ).

The most general quantum state corresponds to a complete graph with all possible multicolored weighted edges between each vertex (see Fig. 2). As an essential step, we need to construct the objective (e.g., state fidelity) in terms of weights, $F(\omega)$. While the entire quantum state $\Phi(\omega)$ is directly defined by the edge weights, conditioning measurements are commonly used to obtain more intricate states and to overcome the lack of single-photon nonlinearities. Prominent examples for such measurements are conditioning on the simultaneous detection of one photon 
in each path (I) or conditioning on the detection of ancilla photons (II).

As an example in Fig. 1, we show the construction of a four-photon Greenberger-Horne-Zeilinger (GHZ) state $|\mathrm{GHZ}\rangle=1 / \sqrt{2}(|0,0,0,0\rangle+|1,1,1,1\rangle)_{a-d}$, where $|0\rangle$ and |1) stand for one photon in the internal mode 0 and 1 (such as horizontal or vertical polarization), respectively. The subscript $a-d$ means one photon is in each of the four paths $a, b, c$, and $d$. Under the condition of simultaneous detection (I), the term $|0,0,0,0\rangle$ can be generated by three different subgraphs: two blue horizontal edges, vertical edges, or crossed edges. The weight of a subgraph is the product of all its edge weights. The weight of the whole term is the sum of all weights of the subgraphs. Therefore, the weight of $|0,0,0,0\rangle$ is

$$
\begin{aligned}
\omega_{|0,0,0,0\rangle} & =\langle 0,0,0,0|\Phi(\omega)| \mathrm{vac}\rangle \\
& =\omega_{a, b}^{0,0} \omega_{c, d}^{0,0}+\omega_{a, c}^{0,0} \omega_{b, d}^{0,0}+\omega_{a, d}^{0,0} \omega_{b, c}^{0,0} .
\end{aligned}
$$

In an equivalent way, the amplitude of $|1,1,1,1\rangle$ can be written in terms of $\omega$. As a result, we have the fidelity written as

$$
F(\omega)=\frac{\left|\omega_{|0,0,0,0\rangle}+\omega_{|1,1,1,1\rangle}\right|^{2}}{2 \cdot N(\omega)^{2}},
$$

where $N(\omega)$ is a normalization constant of the state emerging from the graph (more details in Appendix B).

The weights of the graph are optimized by minimizing a loss function constructed from the fidelity and an additional $L_{1}$ regularization term:

$$
L(\omega)=[1-F(\omega)]+\alpha \cdot|\omega|_{1}
$$

with positive real coefficient $\alpha<1$. Inclusion of the $L_{1}$ regularization term can drive the optimization toward a solution with smaller amplitudes, thereby opening ways to further reduce the edges of the graph by removing edges with small weights. For optimization, we use the BroydenFletcher-Goldfarb-Shanno algorithm, an iterative gradientdescent method that approximates Hessians to solve nonlinear optimization problems. If we identify a solution with $F(\omega)$ above a limit (we use $F_{\text {limit }}=0.95$ ) and small weights $\omega$ (we use $\omega_{\text {limit }}=1$ ), we find a suitable experimental setup candidate. At this point, as the loss minimization is fast, we can perform a topological optimization [50]. We reduce the size of the graph by iteratively removing individual edges. We can choose the edge from a distribution that depends on the magnitude of the weights of the previous solution (with two special cases: choosing entirely randomly and always choosing the edge with the smallest weight magnitude). The new, smaller graph is used to minimize the loss function in Eq. (4). The topological optimization reduces the size of the graph iteratively.
The topological optimization distills small structures such that human scientists can interpret and understand the underlying physical principles and use the new knowledge to solve other cases. In many instances, we use these insights to find straightforward generalizations to infinitely large classes of situations. This ability to generalize is in stark contrast to typical artificial intelligence applications in the natural sciences [51], where the solution of a parameter optimization is the final product, without the intention of discovering new scientific ideas (with few recent exceptions that rediscover previously known physical ideas $[36,37]$ ).

\section{Benchmarking}

We compare the speed of THESEUs with previous approaches, using classes of high-dimensional multipartite states called Schmidt-rank vectors as a benchmark [52]. In particular, we aim to discover maximally entangled threeparty quantum states of up to ten local dimensions. This task is well understood theoretically; thus, it represents a good benchmark. There are 81 unique entangled structures that could be generated using linear optics [45]. A pure topological circuit search using $150 \mathrm{CPU}$ hours has discovered 51 out of 81 different states in the set [20]. A reinforcement learning algorithm has identified 17 out of 81 different states, with speed comparable to the topological search algorithm [24]. THESEUS discovers 76 different states within $2 \mathrm{~h}$, where the first 17 are identified within $2 \mathrm{~s}$ and the first 51 states in less than $15 \mathrm{~min}$. This results in a speed-up of a factor of 600 .

We turn to a second benchmarking task: the identification of high-dimensional CNOT gates. A recent study shows that the identification of the first photonic high-dimensional controlled operation takes $150000 \mathrm{CPU}$ hours [40]. Our algorithm finds a solution that is experimentally quantitatively simpler, within $1 \mathrm{CPU}$ second. This results in a speed-up of a factor $10^{8}$. We come back to this example in Fig. 5.

\section{Scientific discovery and understanding}

The improvement in speed shows that THESEUs is ready to go beyond benchmarks and be applied to the discovery of new scientific targets and to the development of new scientific insights and understanding. Scientific understanding is essential to the epistemic aims of science [53] but rarely addressed in applications of artificial intelligence to the natural sciences. In the philosophy of science, pragmatic criteria have been found for scientific understanding, in particular, by de Regt's award-winning work $[53,54]$. He describes that scientists can understand a phenomenon if they can recognize qualitatively characteristic consequences without performing exact calculations. We connect this criterion to our discoveries: We discover the first high-dimensional six-photonic GHZ states, which have been conjectured to be not constructible with linear optics. We can understand the underlying concept and use it 
to construct a simple method to generate high-dimensional GHZ states with an arbitrary number of photons. Furthermore, we discover the first solutions of heralded three-dimensional Bell states. We also understand the underlying concept, which, among others, contains an idea to destructively interfere vacuum terms. We generalize the concept to arbitrary-dimensional Bell states-without additional calculations. Similarly, we discover setups for heralded GHZ states that need fewer resources than methods proposed in the literature, which could form the building blocks for photonic quantum computation [7]. We furthermore apply THESEUS to multiphotonic transformations. We find a new way to interpret and construct photonic qubit operations such as CNOT gates. Moreover, we discover high-dimensional CNOT operations that need quantitatively fewer resources than methods proposed in the literature. Connecting to de Regt's criterion, our algorithm is the source of scientific understanding in multiple instances.

Why does our approach allow us to understand the solution? An essential substance of the illuminating power is the fact that the final result is presented in a minimal graph, which is the conceptual core of the solution. The final graph can be inspected visually, and visualizability has long been used as a tool to gain understanding. This use goes back at least to Schrödinger, who argued for visualizability (Anschaulichkeit) for intelligible physical theories. Detailed discussions about this tool for understanding can be found in the work of de Regt [53,54]. In this concrete case, the minimal graph represents the quantum state and how it is produced in a visual way. We can detect symmetries or other apparent visual properties that can readily be generalized.

\section{High-dimensional GHZ states}

A $d$-dimensional $n$-partite GHZ quantum state is written as

$$
|\psi\rangle=\frac{1}{\sqrt{d}} \sum_{i=0}^{d-1}|\underbrace{i, i, i, \ldots}_{n \text { times }}\rangle .
$$

These states are studied in the interplay between quantum and local-realistic theories [55] and have recently found potential applications in quantum communication tasks [56].

Graph-theoretical arguments show that perfect highdimensional GHZ states can be generated with standard quantum optical tools (e.g., probabilistic photon-pair sources and postselection) only for four-photon states [41], because terms in addition to those in Eq. (5) necessarily emerge. Using THESEus, we discover the first example that circumvents the no-go theorem; see Fig. 3. The algorithm identifies solutions with fidelities arbitrarily close to one, by adjusting the edge weights such that unwanted terms have arbitrarily small weights (albeit at the (a) Complete 3-dimensional Graph (b) 3-dimensional 6-photonic GHZ state

(c)

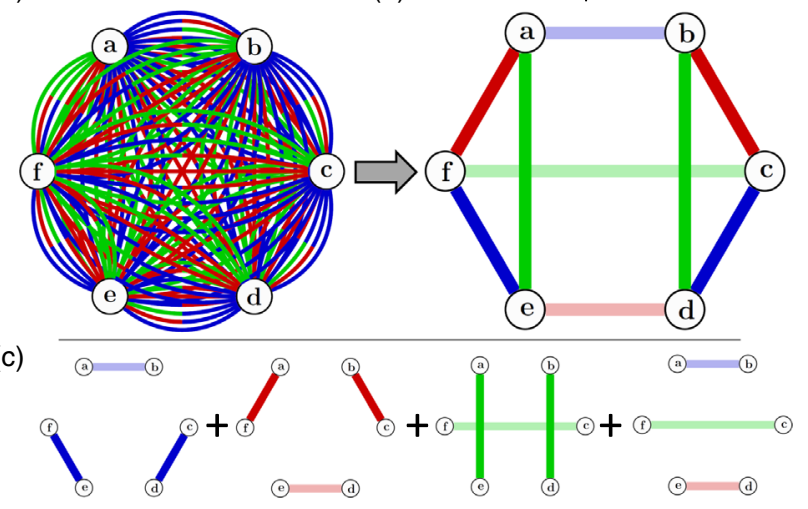

$|\psi\rangle=\omega|0,0,0,0,0,0\rangle+\omega|1,1,1,1,1,1\rangle+\omega|2,2,2,2,2,2\rangle+\omega^{3}|1,1,0,2,2,0\rangle$

FIG. 3. Finding a three-dimensional six-photonic GHZ state. (a) The initial state is a complete graph of six vertices and three colors. Each pair of vertices is connected by nine edges, which stand for all nine possibilities (blue, red, and green stand for modes 0,1 , and 2, respectively). A bicolored edge stands for a photon pair with different mode numbers. For example, a bluered edge between vertices $a$ and $b$ stands for a photon pair with one photon in path $a$ with mode number 0 and one photon in $b$ with mode number 1 , i.e., $a_{0}^{\dagger} b_{1}^{\dagger}$. In total, this procedure leads to 135 edges. (b) The optimized graph for a six-photonic threedimensional GHZ state. While it has been shown that such a state cannot be created with perfect fidelity [41] with linear optics and probabilistic photon-pair sources (without additional photons), THESEUS finds a solution where the fidelity scales with $F \approx 1-$ $O\left(\omega^{4}\right)$ with the overall counts $C$ scaling as $C \approx O\left(\omega^{2}\right)$, which is experimentally feasible. (c) The concept of the solution can be interpreted in the context of graph-theoretical results and can be immediately generalized by human scientists.

expense of lower count rates). The solution can immediately be generalized to GHZ states (and other states) with higher dimensions and a larger number of particles, by identifying subgraphs of additional terms whose edges are multiplied with $\omega<1$.

No further computations or optimizations are necessary, demonstrating that we achieve scientific understanding based on a computational optimization in the appropriate representation of the problem at hand.

\section{Heralded photonic entangled states}

The next targets we address are heralded entangled photonic states. Standard sources of photonic entanglement such as spontaneous parametric down-conversion or spontaneous four-wave mixing are entirely probabilistic [9]. That means photons are produced at random times, and only after the detection of the photon state does one know that they have been created. The generation of heralded states would allow for event-ready schemes, which are essential in photonic quantum computation [7,57]. Experimentally, two-dimensional Bell states have been generated conditioned on the detection of photons in four 

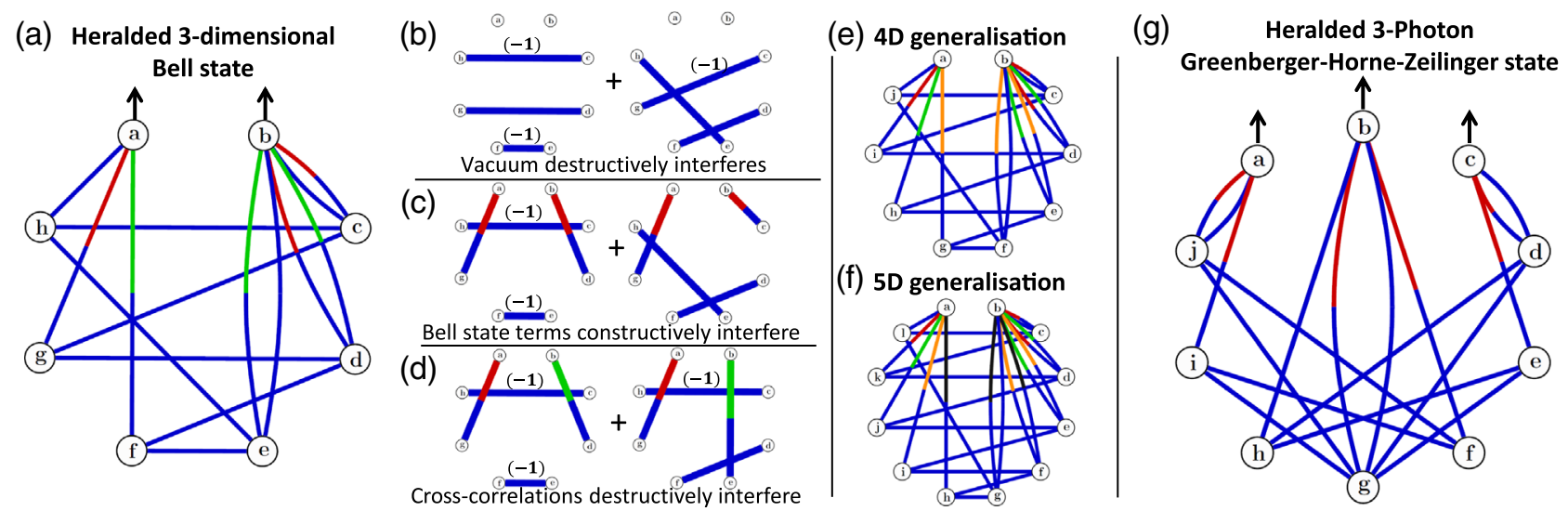

FIG. 4. Heralded entangled states. (a) Optimized graph for a heralded three-dimensionally entangled Bell state with photons in $a$ and $b$ containing a Bell state if detectors in $c-h$ click, which requires eight photons in total. The crucial insight is the destructive interference of the undesired heralded vacuum term. (b) Each of the two subgraphs heralds the vacuum. The overall weight of the two subgraphs differs only in sign, leading to destructive interference. (c) With the same phase setting, all terms of the Bell state constructively interfere, such as $|1,1\rangle$, and all cross-correlation terms cancel, such as $|1,2\rangle$ in (d). This solution can immediately be generalized to arbitrary highdimensional Bell states; see (e) and (f). (g) Solution for a heralded two-dimensional GHZ state in $a-c$, with triggers in $d-j$, requiring ten photons. The algorithm identifies a highly symmetric solution that destructively interferes lower-order eight-photon contributions.

trigger detectors [58,59]. However, higher-dimensional generalizations are missing. A major challenge in creating heralded states are cases where all trigger detectors see a photon, but no photons emerge from the setup. Those cases, where the triggers herald a vacuum term, usually have significantly higher probability of happening than the correct heralded Bell state, because fewer pair creation events need to occur simultaneously.

ThESEus identifies experiments for a heralded threedimensional Bell state; see Fig. 4(a). The setup requires four photon-pair events simultaneously, which is well within today's experimental capabilities [60]. The solution contains a remarkable idea that has not been explored before: the destructive interference of the triggered vacuum term; see Fig. 4(b). Creating the possibility of two heralded vacuum outputs and assigning their amplitudes opposite signs leads to their cancellation.

Furthermore, each of the two subgraphs that lead to a vacuum term in Fig. 4(b) forms the basis of a threedimensional Bell state which constructively interferes, while all cross-correlations destructively interfere. Specifically, two of the three edges are used to herald the trigger detectors, while two additional edges are necessary to create a two-photon output. More information is provided in Supplemental Material [61]. Higher-order events and cases where multiple photons are detected in the trigger detectors can be reduced to arbitrarily low probabilities by adjusting the weights of the edges. Assuming a standard pump laser with $80 \mathrm{MHz}$ repetition rate, the expected count rate to reach a fidelity that guarantees genuine three-dimensional entanglement, i.e., $F>2 / 3$, is on the order of ten per second (for details, see Appendix D). The concepts used by THESEUS, in particular, the cancellation of vacuum, can be immediately generalized to other cases, for example, to arbitrary high-dimensional Bell states; see Figs. 4(e) and 4(f).

Next, we use THESEus to find heralded multiphotonic states, which have been proposed a decade ago but never experimentally implemented due to their experimental requirements $[62,63]$. Heralded GHZ states provide the resources for definite demonstration of deterministic violations of local-realistic world views [64] and are among the most promising building blocks for photonic quantum computation [7,57]. We find an experimental configuration which requires fewer resources and which is within reach of experimental capabilities; see Fig. 4(g). The solution is highly symmetric and uses a very similar concept to avoid lower-order contributions as the solution of the Bell state. In this case, however, the problematic lower-order event creates single-photon outputs. The strategy, again, is to generate two subgraphs for each single-photon output with opposite phase which destructively interfere (see Supplemental Material [61]).

\section{Photonic controlled gates}

Finally, we demonstrate the usage of THESEus for photonic quantum transformations, which are essential elements for photonic quantum simulation [4] and computation schemes [3,17,57]. In Fig. 5, we introduce virtual vertices that represent input photons and optimize multiple dependent graphs simultaneously that represent different states of the transformation. Interestingly, exactly this concept has been the core of one of the first photonic CNOT experiments [65], which gives a new interpretation for a 17-year-old experiment (see Supplemental Material [61] for details).

We apply THESEUS to find high-dimensional quantum transformations, which are discussed in the context of resource-efficient quantum computation algorithms 


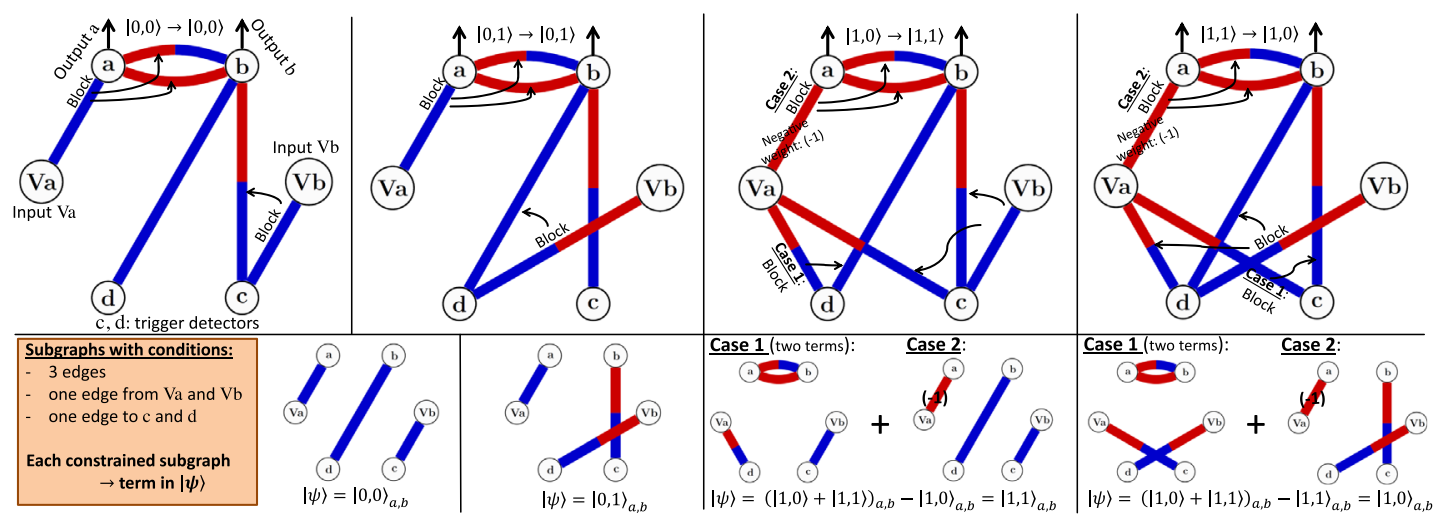

FIG. 5. Qubit CNOT transformation: Two input photons (denoted as Va and Vb) undergo a CNOT operation and exit in path $a$ and $b$, conditioned on the simultaneous detection of one photon in each of the trigger paths $c$ and $d$. This example goes beyond state generation and shows how the same framework allows identification of quantum transformations. We introduce virtual vertices $\mathrm{Va}$ and $\mathrm{Vb}$, which are interpreted as incoming photons. Edges between a virtual vertex and vertices $a-d$ (in all graphs together) represent unitary transformations of the incoming photon. For example, if $\mathrm{Vb}=|0\rangle$, the photon goes to path $c$, while for $\mathrm{Vb}=|1\rangle$ it goes to path $d$. The CNOT consists of four individual transformations (one for each of the inputs $|0,0\rangle,|0,1\rangle,|1,0\rangle$, and $|1,1\rangle$ ). Each transformation stands for one graph, and the subgraph of vertices $a-d$ has to stay constant for each transformation. The four graphs in the upper row are the solution of an automated design for a two-qubit CNOT. The quantum state in the output of $a$ and $b$ (after conditioning on the trigger detectors $c$ and $d$ ) is governed by all subgraphs that fulfill the following conditions (see orange inset): contains three edges (two edges from incoming photons and one ancillary photon pair); each of the virtual vertices $\mathrm{Va}$ and $\mathrm{Vb}$ is contained in one edge (which symbolizes that one photon is entering the setup); and both $c$ and $d$ are contained in an edge (such that the two triggers detect a photon in paths $c$ and $d$ ). The solution can be conveniently interpreted: No vertex can have two incoming edges (as follows from the three conditions). Therefore, an edge involving Va or Vb blocks all edges at the other vertex of the edge, which significantly simplifies the interpretation of the graphs. The resulting terms are written in the lower row. From the solution, we discover an interesting concept: If $\mathrm{Va}=|0\rangle$, the edge involving $\mathrm{Vb}$ chooses the outgoing term by blocking the appropriate edge. However, if $\mathrm{Va}=|1\rangle$, the double edge between $a$ and $b$ is active - as the weight $\omega_{V a, a}^{1,1}=(-1)$, Vb chooses the term that will destructively interfere. The idea of having one virtual vertex choosing the terms can be generalized to more complex multiqubit transformations.

$[66,67]$. The solution follows similar concepts as the twodimensional case and requires fewer experimental resources than Ref. [40]; for details, see Appendix E.

\section{E. Scalability}

In general, it can be extremely challenging to optimize and design experiments for very large quantum systems (a large number of photons), because the state space increases exponentially with the number of photons. This fact is the basis of quantum advantage experiments, both for gatebased quantum computers and for photons via boson sampling. Therefore, one would expect that classical computer algorithms can fundamentally not be scalable design methods for quantum optics. One solution would be to perform the design of quantum optical systems on quantum computers itself [68].

We show another way that allows for the design of large systems using only classical computers. The key is that our algorithm allows us to understand (and thereby generalize) solutions. As we show in Fig. 4, from the computer solution for heralded high-dimensional Bell states, we are able to extract the general concept of how and why they work. Thereby, we are able to generalize the concept to arbitrary dimensional systems. Finding a heralded 50-dimensional Bell state directly with a computer algorithm might be entirely infeasible. However, we find and understand the concept for arbitrary $d$-dimensional Bell states, such that we can write down the solution for a 50-dimensional Bell state immediately.

\section{DISCUSSION}

We present the algorithm THESEUS for the automated design of quantum optical experiments, which is based on an abstract physics-inspired representation. We use it to discover several previously unknown experimental configurations to realize quantum states and transformations in the challenging high-dimensional and multiphotonic regime, such as generation of high-dimensional GHZ states, heralded entangled quantum states, and high-dimensional controlled operations. Those experimental setups are within reach of modern photonic technology and could lead to fascinating experimental investigations of fundamental questions and technological advances. THESEUs can immediately be applied to discover a multitude of other targets in experimental quantum optics, such as tools to enable silicon-photonics quantum computation [57] or highly efficient, low-noise quantum entanglement sources by overcoming the main noise sources in multiphoton experiments [60]. It can also directly be applied to situations where the target state is not known beforehand, 
such as for applications in quantum metrology [2] or in quantum-enhanced microscopes and telescopes [69,70].

From a practical point of view, it is important that the loss function can furthermore be augmented with additional relevant properties and experimental constraints. For example, the expected count rate of the experiment can be optimized simultaneously with the quality of the generated state. This extension is possible because the count rate (as many other properties) of the quantum state can be written in terms of the weights of the graph and could lead to higher count rates of experiments.

The concept behind THESEUS is not restricted to quantum optics but can be applied in a more general setting in physics. In general, the graph-based representation is directly connected to creation and annihilation operators, which are regularly used in quantum physics; thus, THESEUs can further be generalized to a much larger scope. For example, multiexcitation processes (rather than pair excitations as we use here) can be immediately described using hypergraphs [71]. Furthermore, THESEUS could be directly applied to the design of hybrid matterwave-photon systems which use abstractly the same physical description as pure photonic systems. One concrete example involves electron-photon interactions [72]. One further way to directly generalize THESEUs is to take into account Klyshko's advanced-wave picture [73]. In this way, rather than treating an edge in the graph as a photonpair creation, we can interpret it as a quantum information transfer of one single system. This approach will allow us to use THESEUs (including its graph-based representation, the corresponding loss functions, and the ability for topological optimization) for the design of entirely different nonphotonic quantum systems, such as quantum circuits for gate-based quantum computers. We give more details

\section{Generating a 3-qubit GHZ state in a gate-based quantum computer circuit}

(a)

$|0\rangle$

$|0\rangle$

$|0\rangle$

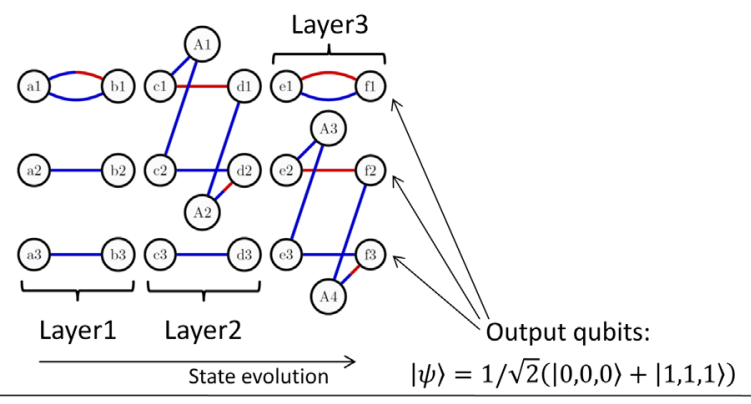

(b)

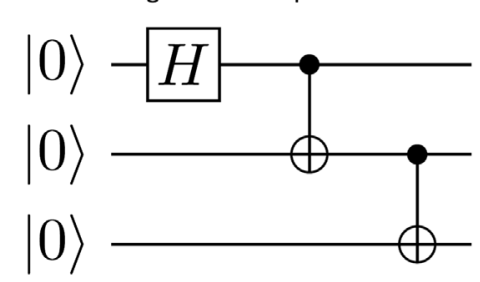

(c)

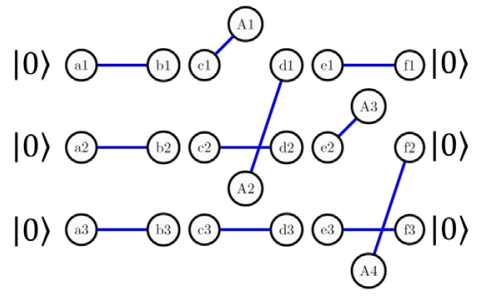

Layerwise joined perfect matchings

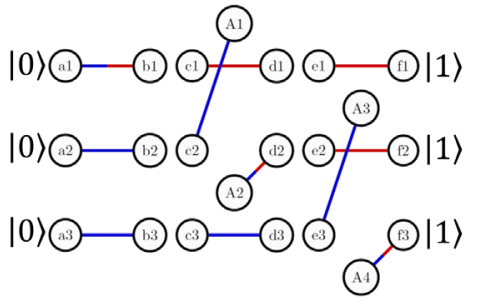

(d)

Potential initial graph for THESEUS

$|0\rangle$

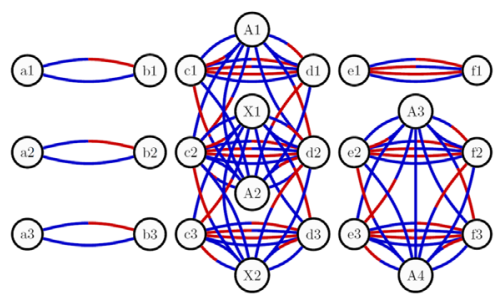

FIG. 6. A quantum circuit for creating a three-qubit GHZ state, represented using graphs that can be optimized by THESEUS. In this concrete case, the graph in (a) represents a quantum circuit that transforms the initial state $|000\rangle$ to a three-qubit GHZ state $1 / \sqrt{2}(|000\rangle+|111\rangle)$ [circuit in (b)]. In (a), every row stands for a qubit, and blue (red) edges stand for $|0\rangle(|1\rangle)$. The vertices $a_{i}$ stand for the input values of the qubit; in this example, all are initialized into $|0\rangle$. The quantum state evolves from left to right. The vertices $b_{i}$ are the final state of layer $1, c_{i}-d_{i}$ and $e_{i}-f_{i}$ are the initial and final quantum information states of the second and third layer, respectively. The vertices $A_{i}$ stand for ancilla vertices to represent control operations between the qubits. The evolution of the state is computed via perfect matchings in the individual layers. The perfect matchings from the layers are then joined; the final state of layer $n$ and the initial state of layer $(n+1)$ need to match. That is because the quantum information cannot change between layers. With this straightforward recipe, we find that there are exactly two joined perfect matchings in the graph, shown in (c). In the left one, all qubits remain in their initial state $|000\rangle$. In the right one, qubit 1 is changed to state $|1\rangle$ in layer 1 ; subsequently, qubit 2 is changed to $|1\rangle$ in layer 2 , and qubit 3 is changed to $|1\rangle$ in the final layer. In this way, the state emerges in $|111\rangle$. As before in the photonic case, the full state is a coherent superposition of all perfect matchings. For that reason, the final state is $1 / \sqrt{2}(|000\rangle+|111\rangle)$, which is a three-qubit GHZ state. This representation can be used for designing nonphotonic gate-based quantum circuits using THESEUS. We start with a general weighted graph, such as (d). Here, qubits can interact with each other only through ancilla vertices; therefore, there are no edges between the qubits from different rows. The first layer allows for single-qubit transformations. The second layer encodes two control operations, one between qubits 1 and 2 and one between 2 and 3. We choose to use only one control operation in layer 3 (to see the allowed structure more clearly). 
for the application in quantum circuit design in Fig. 6 and in Appendix C.

One of the main features is the possibility to extract scientific understanding from computer-inspired designs. This feature is made possible by a topological optimization that reduces the solutions to conceptual cores. Those minimal topologies allow for the interpretation and generalizations of the discovered solution, without performing additional calculations. This ability is in accord with criteria from the philosophy of science that argue that scientific understanding is connected with the skill to use concepts fruitfully, without exact calculations. Hence, in a broader sense, we argue that the ability of our algorithm goes beyond simple optimization and enters the realm of providing scientific insights and allowing for scientific understanding. Thereby-together with its general applicability to large classes of problems in physics-THESEUS directly contributes to scientific, explainable AI and, in general, to an essential aim of science.

Code availability: The source code of THESEUs, including numerous examples, translation of experimental setups to graphs, and graphs to experimental setups can be found on GitHub [74].

\section{ACKNOWLEDGMENTS}

This work was supported by the Google Focused Award on Quantum Computing, the Industrial Research Chair Program of Canada, and the U.S. Department of Energy, Office of Science, Office of Advanced Scientific Computing Research, Quantum Algorithm Teams Program. A. A.-G. acknowledges generous support from the Canada 150 Research Chair Program, Tata Steel, Anders G. Frøseth, the Office of Naval Research. M. K. acknowledges support from the Austrian Science Fund (FWF) through the Erwin Schrödinger fellowship No. J4309. N. T. acknowledges support by the Griffith University Postdoctoral Fellowship
Scheme, the Australian Research Council (ARC) Centre of Excellence CE170100012, and by the Alexander von Humboldt Foundation.

\section{APPENDIX A: TRANSLATION TO EXPERIMENT}

In Figs. 7 and 8, we show how to translate subgraphs to different experimental configurations. In Fig. 9, we show how to translate one graph to a number of different quantum experiments.

\section{APPENDIX B: NORMALIZATION OF QUANTUM STATES}

We show how the state of a complete two-colored graph with four vertices can be written using the weight function $\Phi(\omega)$ of a graph. It can be represented in terms of creation operators as

$\Phi(\omega) \approx \sum_{n} \frac{1}{n !}\left(\sum_{\substack{x, y \in\{a, b, c, d\} \\ x<y}} \sum_{c_{1}, c_{2} \in\{0,1\}} \omega_{x, y}^{c_{1}, c_{2}} x_{c_{1}}^{\dagger} y_{c_{2}}^{\dagger}+\text { H.c. }\right)^{n}$.

If we are conditioning the state on one photon in each detector, it reduces to

$$
|\psi\rangle=\frac{1}{N(\omega)} \sum_{i, j, k, l \in\{0,1\}} \omega_{|i, j, k, l\rangle}|i, j, k, l\rangle
$$

with the edge weights

$$
\begin{aligned}
\omega_{|i, j, k, l\rangle} & =\langle i, j, k, l|\Phi(\omega)| \mathrm{vac}\rangle \\
& =\omega_{a, b}^{i, j} \cdot \omega_{c, d}^{k, l}+\omega_{a, c}^{i, k} \cdot \omega_{b, d}^{j, l}+\omega_{a, d}^{i, l} \cdot \omega_{b, c}^{j, k}
\end{aligned}
$$

and the normalization constant

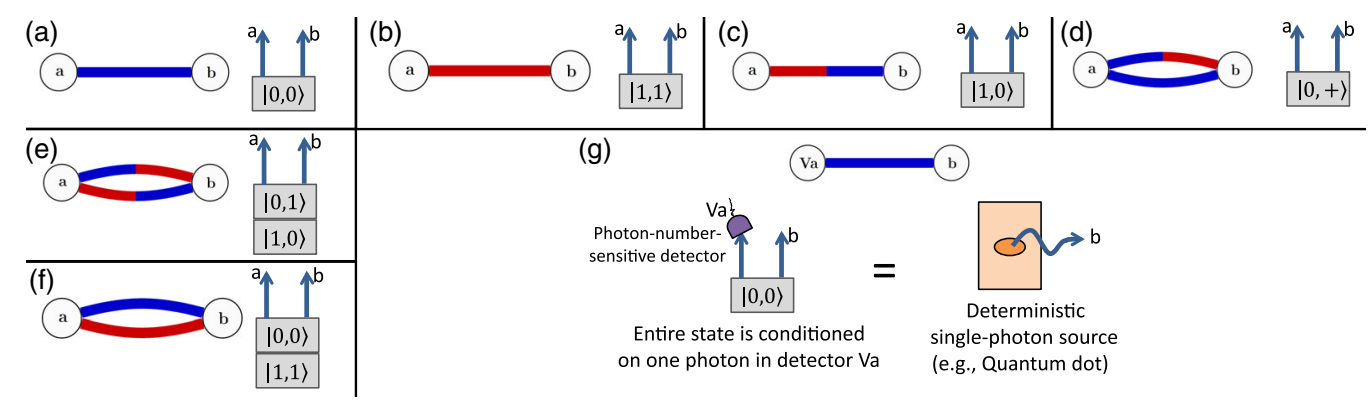

FIG. 7. Graph to experiment translation for individual edges. Designing quantum optical experiments using the abstract notation of graphs is possible because we find translations of graphs into several different experimental schemes. Edges between vertices $a$ and $b$ are translated to probabilistic photon sources; see (a)-(f). Edge colors correspond to mode numbers. Multiedges correspond to superposition or entanglement and can be created with standard photonic technologies, for example, cross-crystal sources [75,76]. (g) A deterministic single-photon source emitting in path $b$ can be understood as an edge between a vertex $b$ and a virtual vertex Va. For each term in the resulting quantum state, every virtual vertex always needs to have exactly one incoming edge. This constraint is conceptually equivalent to the situation of a probabilistic photon-pair source, where the whole state is conditioned on the detection of one photon using a photonnumber-sensitive detector in path Va. 


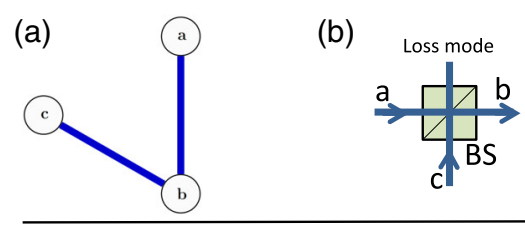

(f)

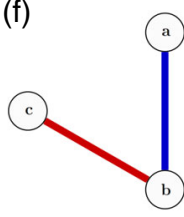

(c)
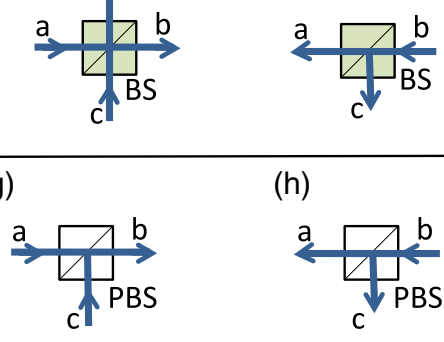

(h)

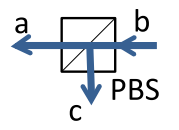

(d)
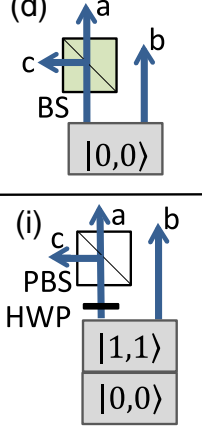

(e)

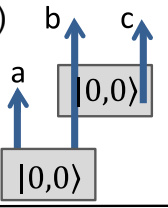

(j)

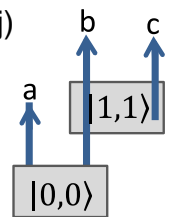

FIG. 8. Merging edges of graphs. Edges can be merged at one vertex in several different ways. (a) If the edges have the same color, the corresponding photons have the same mode number. (b)-(d) In that case, the edges can be merged with probabilistic beam splitters (green squares) or by creating them directly with path identified photon-pair sources [for instance, SPDC crystals; see (e)]. (f) If the edges have different colors, the corresponding photons have different mode numbers. In that case, the edges can be merged losslessly with mode-dependent beam splitters (so-called multiplexing or demultiplexing); white squares, for example, polarizing beam splitters if the degree of freedom is photonic polarization [see (g)-(i)]. (j) The edges could also be created by path identified photon-pair sources (for instance, SPDC crystals). Other probabilistic photon sources, such as lasers as probabilistic single-photon sources, can be added by exploiting hypergraph structures [71].

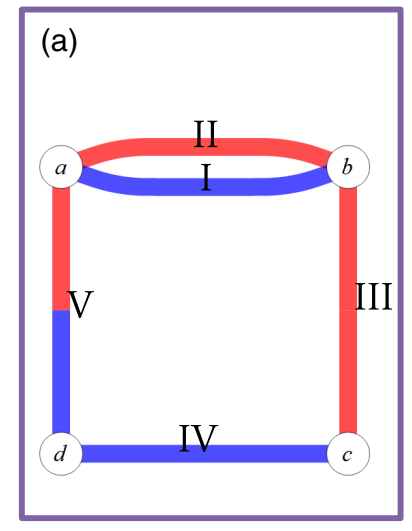

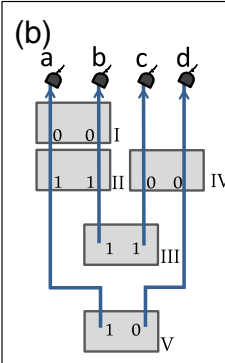

Entanglement by path identity (c)

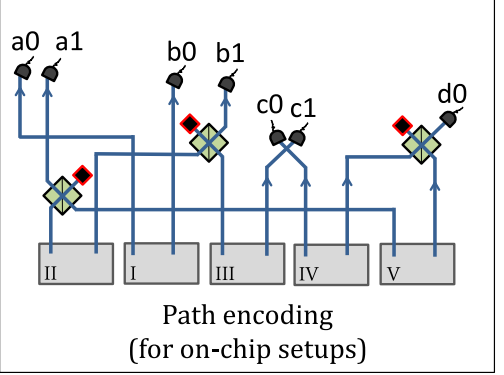

(d)

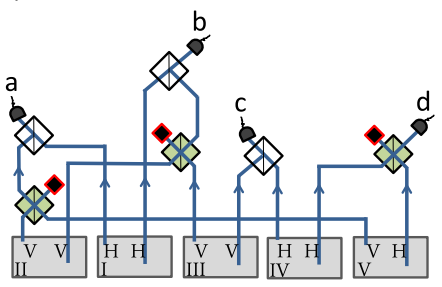

Polarization entanglement (for bulk optics)

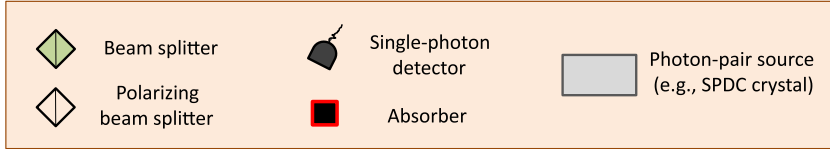

FIG. 9. Translation of a graph to three different types of quantum optics experiments. (a) A graph with four vertices and five edges and two colors represents a four-photon quantum experiment using two state levels (dimensions), such as polarization. (b) Implementation using entanglement by path identity. (c) Implementation using path encoding, which is the most commonly used encoding for on-chip quantum optics experiments. (d) Implementation using bulk optics for polarization quantum optics experiments.

$$
N(\omega)=\sqrt{\sum_{i, j, k, l \in\{0,1\}}\left|\omega_{\mid i, j, k, l}\right|^{2}} .
$$

The objective of the optimization is to find $\omega_{x, y}^{i, j} \in \mathbb{C}$ that minimize the loss function and, subsequently, find solutions with a large number of edge weights being zero. The information about higher-order contributions to the state, which results in experimentally reduced quantum fidelities, is encoded within the weight function $\Phi(\omega)$. Therefore, higher-order contributions could be directly accounted for within the optimization procedure. More details about the approximations in Eq. (B1) can be found in Refs. [77,78].

\section{APPENDIX C: QUANTUM CIRCUIT DESIGN}

In 1994, Belinskii and Klyshko describe "an intuitive treatment of two-photon correlation with the help of the concept of an effective field acting upon one of the two detectors and formed by parametric conversion of the advanced wave emitted by the second detector" [73]. Let us now imagine a situation where a nonlinear crystal produces two photons, detected by detector $D 1$, the other one in detector D2. Belinskii and Klyshko interpret this situation in a different way. They imagine a photon being produced at the place of detector D1, which travels back to the crystal. At the crystal, it is reflected and travels toward detector $D 2$. This picture allows for the 
interpretation of numerous quantum correlation effects. Importantly, it gives an intuition that the representation of THESEUS can not only describe SPDC crystals but abstract quantum information flow. This significantly expands the field of application of THESEUS to nonphotonic quantum systems. We demonstrate this intuition in a concrete nonphotonic example, namely, the design of quantum circuits for gate-based quantum computers.

The loss function can be written in terms of the weights of the graph, entirely analogously to the photonic examples in the main text. That is because the evolution of the state can be represented as weights $\omega$, and the emerging quantum state and all of its properties (such as the state fidelity at the end of the quantum circuit) can be written as a function of the weights $\omega$. In this way, THESEus can perform gradient-based optimization of the weights in the graph. Furthermore, the topological optimization removes edges and, thereby, simplifies the final quantum circuit.

To translate the graph to quantum gates, we can simply inspect the action at every individual level. For example, in Fig. 6 in the first layer 1, we have two perfect matchings $\left(a_{1}, b_{1}, a_{2}, b_{2}, a_{3}, b_{3}\right)=(r, r, r, r, r, r)$ and $(r, g, r, r, r, r)$. These perfect matchings mean the state evolves from $|000\rangle$ to $1 / \sqrt{2}(|0\rangle+|1\rangle)|00\rangle$. The action of this transformation resembles exactly that of Hadamard operations. In a similar way, we can analyze the action of the input-output relation of every layer, and individual layers can easily be rewritten in terms of universal gates. A detailed analysis goes far beyond the scope of this manuscript. This example, however, shows that Theseus and its representation and the optimization algorithm are not restricted to quantum optics but can represent and solve a wide variety of tasks in physics and quantum physics.

For the future, it will be very interesting to identify similarities and differences between this application of THESEUS and other quantum circuit design algorithms. In particular, it will be very interesting to compare other graphbased design principles such as the ZX formalism [79].

Again, at a big-picture level, this example shows that the graph representation, the weight function, and the gradientbased optimization are not restricted to the design of quantum optics experiments but can also be useful in the design of quantum information processes or potentially quantum logic design.

\section{APPENDIX D: FIDELITY AND COUNT RATE OF A HERALDED THREE-DIMENSIONAL BELL STATE}

The fidelity of the three-dimensional Bell state can be arbitrary close to one, by adjusting the weights of the edges. In the most straightforward setting, all edges that are connected to $a$ or $b$ have the same weight $v$, while all edges connecting ancilla vertices $c-h$ have weight $w$ (with phases as shown in the main text). In this way, the heralded state can be written as
TABLE I. Fidelity and count rates for heralded three-dimensional Bell states.

\begin{tabular}{llcc}
\hline \hline$v$ & \multicolumn{1}{c}{$w$} & Fidelity & Count rate \\
\hline 0.16 & 0.07 & $2 / 3$ & $18.8 \mathrm{~Hz}$ \\
0.125 & 0.048 & 0.75 & $1.5 \mathrm{~Hz}$ \\
0.1 & 0.035 & 0.8 & $0.8 \mathrm{~Hz}$ \\
0.0820219 & 0.0240018 & 0.85 & 65 per hour \\
0.0576405 & 0.0139269 & 0.9 & 1.8 per hour \\
\hline \hline
\end{tabular}

$$
\begin{aligned}
|\psi\rangle= & 2 v^{2} w^{2}(|0,0\rangle-|1,1\rangle-|2,2\rangle)_{a, b} \\
& +v w^{3}|\phi\rangle_{\text {one photon }}+w^{4}|\phi\rangle_{\text {zero photons }} \\
& +\mathcal{O} \text { (higher orders) }
\end{aligned}
$$

where $|\phi\rangle_{\text {one photon }}$ stands for combinations where three ancilla photon pairs and one pair containing an ancilla photon and an output photon are produced. The state $|\phi\rangle_{\text {zero photon }}$ are cases where four ancillary photon pairs are created. Both of those terms can be reduced by making $w$ smaller than $v$. The term $\mathcal{O}$ (higher orders) correspond to cases with five or more photon pairs produced, which can be reduced by having $v$ and $w$ smaller than one.

We calculate the fidelity and expected count rates for various settings of weights $v$ and $w$ in Table I, calculated up to the sixth order of SPDC, and not considering any losses or detector inefficiency.

\section{APPENDIX E: CNOT BEYOND QUBITS}

A control operation in a $2 \times 3$-dimensional space is shown in Fig. 10. The subgraphs $a-f$ remain constant, while the edges containing $\mathrm{Va}$ and $\mathrm{Vb}$ change depending on the input control or target photons. The correct transformation is heralded by simultaneous detection of a photon in each of the detectors $c-f$. The structure of the subgraphs $a-f$ is very reminiscent of the solution of
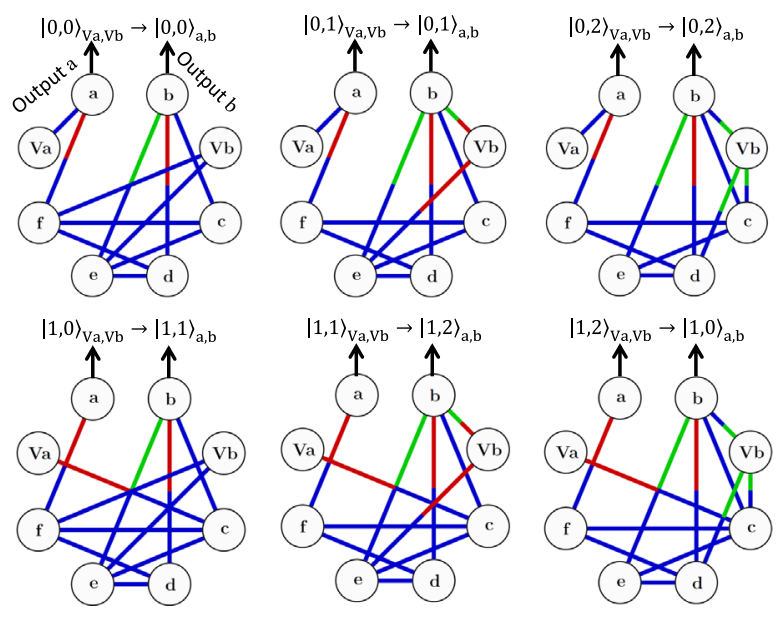

FIG. 10. High-dimensional CNOT gate, with a qubit control photon and a qutrit target photon. 
heralded Bell states in Fig. 4 in the main text. Here, each internal mode (represented as edge color) from $a$ and $b$ is connected to one individual heralding detector.

Furthermore, the solution uses destructive interference for producing the correct output states, as in Fig. 4 in the main text. Some of the resulting subgraphs (those that have one incoming edge to vertex $c-f$ ) do not vanish. Still, they are reduced in magnitude by adapting the edge weights appropriately. Thereby, an experimentally feasible method of performing CNOT transformations beyond qubits is constructed.

[1] P.-A. Moreau, E. Toninelli, T. Gregory, and M. J. Padgett, Imaging with Quantum States of Light, Nat. Rev. Phys. 1, 367 (2019).

[2] E. Polino, M. Valeri, N. Spagnolo, and F. Sciarrino, Photonic Quantum Metrology, AVS Quantum Sci. 2, 024703 (2020).

[3] F. Flamini, N. Spagnolo, and F. Sciarrino, Photonic Quantum Information Processing: A Review, Rep. Prog. Phys. 82, 016001 (2018).

[4] A. Aspuru-Guzik and P. Walther, Photonic Quantum Simulators, Nat. Phys. 8, 285 (2012).

[5] S. Aaronson and A. Arkhipov, The Computational Complexity of Linear Optics, in Proceedings of the Forty-Third Annual ACM Symposium on Theory of Computing, San Jose, CA (Association for Computing Machinery, New York, 2011), pp. 333-342.

[6] A. Peruzzo, J. McClean, P. Shadbolt, M.-H. Yung, X.-Q. Zhou, P. J. Love, A. Aspuru-Guzik, and J. L. O'Brien, A Variational Eigenvalue Solver on a Photonic Quantum Processor, Nat. Commun. 5, 4213 (2014).

[7] M. Gimeno-Segovia, P. Shadbolt, D. E. Browne, and T. Rudolph, From Three-Photon Greenberger-HorneZeilinger States to Ballistic Universal Quantum Computation, Phys. Rev. Lett. 115, 020502 (2015).

[8] J. Carolan, C. Harrold, C. Sparrow, E. Martín-López, N. J. Russell, J. W. Silverstone, P. J. Shadbolt, N. Matsuda, M. Oguma, M. Itoh et al., Universal Linear Optics, Science 349, 711 (2015).

[9] J. Wang, F. Sciarrino, A. Laing, and M. G. Thompson, Integrated Photonic Quantum Technologies, Nat. Photonics 14, 273 (2020).

[10] L.-T. Feng, G.-C. Guo, and X.-F. Ren, Progress on Integrated Quantum Photonic Sources with Silicon, Adv. Quantum Technol. 3, 1900058 (2019).

[11] D. Llewellyn, Y. Ding, I. I. Faruque, S. Paesani, D. Bacco, R. Santagati, Y.-J. Qian, Y. Li, Y.-F. Xiao, M. Huber et al., Chip-to-Chip Quantum Teleportation and Multi-Photon Entanglement in Silicon, Nat. Phys. 16, 148 (2020).

[12] L. Lu, L. Xia, Z. Chen, L. Chen, T. Yu, T. Tao, W. Ma, Y. Pan, X. Cai, Y. Lu et al., Three-Dimensional Entanglement on a Silicon Chip, npj Quantum Inf. 6, 30 (2020).

[13] H. Rubinsztein-Dunlop, A. Forbes, M. V. Berry, M. R. Dennis, D. L. Andrews, M. Mansuripur, C. Denz, C. Alpmann, P. Banzer, T. Bauer et al., Roadmap on Structured Light, J. Opt. 19, 013001 (2017).
[14] X.-L. Wang, Y.-H. Luo, H.-L. Huang, M.-C. Chen, Z.-E. Su, C. Liu, C. Chen, W. Li, Y.-Q. Fang, X. Jiang et al., 18-Qubit Entanglement with Six Photons' Three Degrees of Freedom, Phys. Rev. Lett. 120, 260502 (2018).

[15] Y.-H. Luo, H.-S. Zhong, M. Erhard, X.-L. Wang, L.-C. Peng, M. Krenn, X. Jiang, L. Li, N.-L. Liu, C.-Y. Lu et al., Quantum Teleportation in High Dimensions, Phys. Rev. Lett. 123, 070505 (2019).

[16] H. Wang, Y. He, Y.-H. Li, Z.-E. Su, B. Li, H.-L. Huang, X. Ding, M.-C. Chen, C. Liu, J. Qin et al., High-Efficiency Multiphoton Boson Sampling, Nat. Photonics 11, 361 (2017).

[17] S. Slussarenko and G. J. Pryde, Photonic Quantum Information Processing: A Concise Review, Appl. Phys. Rev. 6, 041303 (2019).

[18] N. M. VanMeter, P. Lougovski, D. B. Uskov, K. Kieling, J. Eisert, and J. P. Dowling, General Linear-Optical Quantum State Generation Scheme: Applications to Maximally PathEntangled States, Phys. Rev. A 76, 063808 (2007).

[19] M. Krenn, M. Erhard, and A. Zeilinger, Computer-Inspired Quantum Experiments, Nat. Rev. Phys. 2, 649 (2020).

[20] M. Krenn, M. Malik, R. Fickler, R. Lapkiewicz, and A. Zeilinger, Automated Search for New Quantum Experiments, Phys. Rev. Lett. 116, 090405 (2016).

[21] X. Zhan, K. Wang, L. Xiao, Z. Bian, Y. Zhang, B. C. Sanders, C. Zhang, and P. Xue, Experimental Quantum Cloning in a Pseudo-Unitary System, Phys. Rev. A 101, 010302(R) (2020).

[22] P. A. Knott, A Search Algorithm for Quantum State Engineering and Metrology, New J. Phys. 18, 073033 (2016).

[23] R. Nichols, L. Mineh, J. Rubio, J. C. F. Matthews, and P. A. Knott, Designing Quantum Experiments with a Genetic Algorithm, Quantum Sci. Technol. 4, 045012 (2019).

[24] A. A. Melnikov, H. P. Nautrup, M. Krenn, V. Dunjko, M. Tiersch, A. Zeilinger, and H. J. Briegel, Active Learning Machine Learns to Create New Quantum Experiments, Proc. Natl. Acad. Sci. U.S.A. 115, 1221 (2018).

[25] J. M. Arrazola, T. R. Bromley, J. Izaac, C. R. Myers, K. Brádler, and N. Killoran, Machine Learning Method for State Preparation and Gate Synthesis on Photonic Quantum Computers, Quantum Sci. Technol. 4, 024004 (2019).

[26] R. D. King, J. Rowland, S. G. Oliver, M. Young, W. Aubrey, E. Byrne, M. Liakata, M. Markham, P. Pir, L. N. Soldatova et al., The Automation of Science, Science 324, 85 (2009).

[27] M. Schmidt and H. Lipson, Distilling Free-Form Natural Laws from Experimental Data, Science 324, 81 (2009).

[28] B. Sanchez-Lengeling and A. Aspuru-Guzik, Inverse Molecular Design Using Machine Learning: Generative Models for Matter Engineering, Science 361, 360 (2018).

[29] P. S. Gromski, A. B. Henson, J. M. Granda, and L. Cronin, How to Explore Chemical Space Using Algorithms and Automation, Nat. Rev. Chem. 3, 119 (2019).

[30] R. Pollice, G. P. Gomes, M. Aldeghi, R. J. Hickman, M. Krenn, C. Lavigne, M. Lindner-D’Addario, A. Nigam, C. T. Ser, Z. Yao, and A. Aspuru-Guzik, Data-Driven Strategies for Accelerated Materials Design, Acc. Chem. Res. 54, 849 (2021).

[31] S. Molesky, Z. Lin, A. Y. Piggott, W. Jin, J. Vucković, and A. W. Rodriguez, Inverse Design in Nanophotonics, Nat. Photonics 12, 659 (2018). 
[32] W. Ma, Z. Liu, Z. A. Kudyshev, A. Boltasseva, W. Cai, and Y. Liu, Deep Learning for the Design of Photonic Structures, Nat. Photonics 15, 77 (2021).

[33] T. Fösel, P. Tighineanu, T. Weiss, and F. Marquardt, Reinforcement Learning with Neural Networks for Quantum Feedback, Phys. Rev. X 8, 031084 (2018).

[34] S. Sim, P. D. Johnson, and A. Aspuru-Guzik, Expressibility and Entangling Capability of Parameterized Quantum Circuits for Hybrid Quantum-Classical Algorithms, Adv. Quantum Technol. 2, 1900070 (2019).

[35] R. Roscher, B. Bohn, M. F. Duarte, and J. Garcke, Explainable Machine Learning for Scientific Insights and Discoveries, IEEE Access 8, 42200 (2020).

[36] R. Iten, T. Metger, H. Wilming, L. del Rio, and R. Renner, Discovering Physical Concepts with Neural Networks, Phys. Rev. Lett. 124, 010508 (2020).

[37] A. Seif, M. Hafezi, and C. Jarzynski, Machine Learning the Thermodynamic Arrow of Time, Nat. Phys. 17, 105 (2020).

[38] M. Cranmer, A. Sanchez-Gonzalez, P. Battaglia, R. Xu, K. Cranmer, D. Spergel, and S. Ho, Discovering Symbolic Models from Deep Learning with Inductive Biases, arXiv: 2006.11287.

[39] M. Krenn, A. Hochrainer, M. Lahiri, and A. Zeilinger, Entanglement by Path Identity, Phys. Rev. Lett. 118, 080401 (2017).

[40] X. Gao, M. Erhard, A. Zeilinger, and M. Krenn, ComputerInspired Concept for High-Dimensional Multipartite Quantum Gates, Phys. Rev. Lett. 125, 050501 (2020).

[41] M. Krenn, X. Gu, and A. Zeilinger, Quantum Experiments and Graphs: Multiparty States as Coherent Superpositions of Perfect Matchings, Phys. Rev. Lett. 119, 240403 (2017).

[42] X. Gu, M. Erhard, A. Zeilinger, and M. Krenn, Quantum Experiments and Graphs II: Quantum Interference, Computation, and State Generation, Proc. Natl. Acad. Sci. U.S.A. 116, 4147 (2019).

[43] P. G. Kwiat, E. Waks, A. G. White, I. Appelbaum, and P. H. Eberhard, Ultrabright Source of Polarization-Entangled Photons, Phys. Rev. A 60, R773 (1999).

[44] J.-W. Pan, Z.-B. Chen, C.-Y. Lu, H. Weinfurter, A. Zeilinger, and M. Żukowski, Multiphoton Entanglement and Interferometry, Rev. Mod. Phys. 84, 777 (2012).

[45] X. Gu, L. Chen, A. Zeilinger, and M. Krenn, Quantum Experiments and Graphs. III. High-Dimensional and Multiparticle Entanglement, Phys. Rev. A 99, 032338 (2019).

[46] X.-L. Wang, L.-K. Chen, W. Li, H.-L. Huang, C. Liu, C. Chen, Y.-H. Luo, Z.-E. Su, D. Wu, Z.-D. Li et al., Experimental Ten-Photon Entanglement, Phys. Rev. Lett. 117, 210502 (2016).

[47] C. Schaeff, R. Polster, M. Huber, S. Ramelow, and A. Zeilinger, Experimental Access to Higher-Dimensional Entangled Quantum Systems Using Integrated Optics, Optica 2, 523 (2015).

[48] J. Kysela, M. Erhard, A. Hochrainer, M. Krenn, and A. Zeilinger, Path Identity as a Source of High-Dimensional Entanglement, Proc. Natl. Acad. Sci. U.S.A. 117, 26118 (2020).

[49] L.-T. Feng, M. Zhang, D. Liu, Y.-J. Cheng, G.-P. Guo, D.-X. Dai, G.-C. Guo, M. Krenn, and X.-F. Ren, Observation of Nonlocal Quantum Interference between the Origins of a Four-Photon State in a Silicon Chip, arXiv:2103.14277.
[50] Topological optimization is the simplification of the graph or network topology. Put simply, the topological optimization simplifies the graph that represents the quantum experiment by removing edges in the graph.

[51] G. Carleo, I. Cirac, K. Cranmer, L. Daudet, M. Schuld, N. Tishby, L. Vogt-Maranto, and L. Zdeborová, Machine Learning and the Physical Sciences, Rev. Mod. Phys. 91, 045002 (2019).

[52] M. Huber and J. I. Vicente, Structure of Multidimensional Entanglement in Multipartite Systems, Phys. Rev. Lett. 110, 030501 (2013).

[53] H. W. de Regt and D. Dieks, A Contextual Approach to Scientific Understanding, Synthese 144, 137 (2005).

[54] H.W. de Regt, Understanding Scientific Understanding (Oxford University, New York, 2017).

[55] J. Lawrence, Rotational Covariance and GreenbergerHorne-Zeilinger Theorems for Three or More Particles of Any Dimension, Phys. Rev. A 89, 012105 (2014).

[56] X.-M. Hu, W.-B. Xing, C. Zhang, B.-H. Liu, M. Pivoluska, M. Huber, Y.-F. Huang, C.-F. Li, and G.-C. Guo, Experimental Creation of Multi-Photon High-Dimensional Layered Quantum States, npj Quantum Inf. 6, 88 (2020).

[57] T. Rudolph, Why I Am Optimistic about the SiliconPhotonic Route to Quantum Computing, APL Photonics 2, 030901 (2017).

[58] S. Barz, G. Cronenberg, A. Zeilinger, and P. Walther, Heralded Generation of Entangled Photon Pairs, Nat. Photonics 4, 553 (2010).

[59] C. Wagenknecht, C.-M. Li, A. Reingruber, X.-H. Bao, A. Goebel, Y.-A. Chen, Q. Zhang, K. Chen, and J.-W. Pan, Experimental Demonstration of a Heralded Entanglement Source, Nat. Photonics 4, 549 (2010).

[60] H.-S. Zhong, Y. Li, W. Li, L.-C. Peng, Z.-E. Su, Y. Hu, Y.-M. He, X. Ding, W. Zhang, H. Li et al., 12-Photon Entanglement and Scalable Scattershot Boson Sampling with Optimal Entangled-Photon Pairs from Parametric Down-Conversion, Phys. Rev. Lett. 121, 250505 (2018).

[61] See Supplemental Material at http://link.aps.org/ supplemental/10.1103/PhysRevX.11.031044 for additional information on heralded entangled states and the graph of an experimental 2-qubit CNOT gate.

[62] P. Walther, M. Aspelmeyer, and A. Zeilinger, Heralded Generation of Multiphoton Entanglement, Phys. Rev. A 75, 012313 (2007).

[63] X.-L. Niu, Y.-X. Gong, X.-B. Zou, Y.-F. Huang, and G.-C. Guo, Heralded Multiphoton GHZ-Type Polarization Entanglement Generation from Parametric Down-Conversion Sources, J. Mod. Opt. 56, 936 (2009).

[64] C. Erven, E. Meyer-Scott, K. Fisher, J. Lavoie, B. L. Higgins, Z. Yan, C. J. Pugh, J.-P. Bourgoin, R. Prevedel, L. K. Shalm et al., Experimental Three-Photon Quantum Nonlocality under Strict Locality Conditions, Nat. Photonics 8, 292 (2014).

[65] S. Gasparoni, J.-W. Pan, P. Walther, T. Rudolph, and A. Zeilinger, Realization of a Photonic Controlled-NOT Gate Sufficient for Quantum Computation, Phys. Rev. Lett. 93, 020504 (2004).

[66] A. Bocharov, M. Roetteler, and K. M. Svore, Factoring with Qutrits: Shor's Algorithm on Ternary and Metaplectic Quantum Architectures, Phys. Rev. A 96, 012306 (2017). 
[67] M. Jafarzadeh, Y.-D. Wu, Y. R. Sanders, and B. C. Sanders, Randomized Benchmarking for Qudit Clifford Gates, New J. Phys. 22, 063014 (2020).

[68] J. Kottmann, M. Krenn, T. H. Kyaw, S. Alperin-Lea, and A. Aspuru-Guzik, Quantum Computer-Aided Design of Quantum Optics Hardware, Quantum Sci. Technol. (to be published).

[69] C. Parazzoli, B. Koltenbah, D. Gerwe, P. Idell, B. Gard, R. Birrittella, S. Rafsanjani, M. Mirhosseini, O. Magan-Loiza, J. Dowling et al., Enhanced Thermal Object Imaging by Photon Addition or Subtraction, arXiv:1609.02780.

[70] S. M. H. Rafsanjani, M. Mirhosseini, O. S. Magaña-Loaiza, B. T. Gard, R. Birrittella, B. E. Koltenbah, C. G. Parazzoli, B. A. Capron, C. C. Gerry, J. P. Dowling et al., QuantumEnhanced Interferometry with Weak Thermal Light, Optica 4, 487 (2017).

[71] X. Gu, L. Chen, and M. Krenn, Quantum Experiments and Hypergraphs: Multiphoton Sources for Quantum Interference, Quantum Computation, and Quantum Entanglement, Phys. Rev. A 101, 033816 (2020).

[72] O. Kfir, Entanglements of Electrons and Cavity Photons in the Strong-Coupling Regime, Phys. Rev. Lett. 123, 103602 (2019).
[73] A. V. Belinskii and D. N. Klyshko, Two-Photon Optics: Diffraction, Holography, and Transformation of Two-Dimensional Signals, J. Exp. Theor. Phys. 78, 259 (1994).

[74] https://github.com/aspuru-guzik-group/Theseus.

[75] L. Hardy, Source of Photons with Correlated Polarisations and Correlated Directions, Phys. Lett. A 161, 326 (1992).

[76] P. G. Kwiat, K. Mattle, H. Weinfurter, A. Zeilinger, A. V. Sergienko, and Y. Shih, New High-Intensity Source of Polarization-Entangled Photon Pairs, Phys. Rev. Lett. 75, 4337 (1995).

[77] X. Y. Zou, L. J. Wang, and L. Mandel, Induced Coherence and Indistinguishability in Optical Interference, Phys. Rev. Lett. 67, 318 (1991).

[78] S. P. Walborn, C. H. Monken, S. Pádua, and P. H. Souto Ribeiro, Spatial Correlations in Parametric Down-Conversion, Phys. Rep. 495, 87 (2010).

[79] B. Coecke, D. Horsman, A. Kissinger, and Q. Wang, Kindergarden Quantum Mechanics Graduates (... or How I Learned to Stop Gluing LEGO Together and Love the ZX-Calculus), arXiv:2102.10984. 